\title{
Craniofacial growth and development of Turner syndrome children
}

\author{
Inne Suherna Sasmita*, Arlette Suzy Puspa Pertiwi*, M. Harun Achmad** \\ *Department of Pediatric Dentistry Faculty of Dentistry Universitas Padjadjaran \\ ${ }^{* *}$ Department of Pediatric Dentistry Faculty of Dentistry Universitas Hasanudin
}

\begin{abstract}
Turner syndrome is a genetic disorder which characterized by specific physical appearance and the lost of one of sex chromosome in females. The most frequents chromosome constitution in Turner syndrome is 45X. This disorder may cause an interruption of growth and development in the whole body as well as in the craniofacial region. The oral manifestations of Turner syndrome are micrognathia, high palate, malocclusion, and premature eruption of first permanent molars. This paper will discuss the oral manifestations associated with the craniofacial growth and development of Turner syndrome.
\end{abstract}

Key words: Turner syndrome, sex chromosome, craniofacial

\section{INTRODUCTION}

Turner syndrome is a genetic disorder in female which characterized by special physical features and the lost of all or a part of secondary sex chromosome. The disorder may lead to a group of physical finding including congenital lymphoedema, short stature, and gonadal dysgenesis. ${ }^{1,2}$

History of Turner syndrome begun at 1938 when Henry Turner, an endocrinologist from Oklahoma, found seven patients age about fifteen to twenty three came with a problem of dwarfism and delayed sexual development. Patient did not respond to the therapy with pituitary extract. ${ }^{3}$

Incidence of Turner syndrome is 1:20002500 live birth female. It is a rare disorder and characterized by decreased sexual development at puberty. Other features are abnormalities in the heart, kidneys, and other malformations since the syndrome affects many system organ. ${ }^{3,4}$ Increase of chromosome abnormality commonly occurs in children who are born from parents with advanced age, but this does not occur in turner syndrome cases. Main cause of the lost of all or a part of X-chromosome is unknown (Fig. 1). ${ }^{5}$ Short stature is a common sign of Turner syndrome and occurs almost $100 \%$ of cases. Short stature may cause an increase of unbalance body weight, which may lead to a high risk of obesity related disease. Growth deficiency occurs especially in lower extremities as oppose to upper resulted a short feet appearance. ${ }^{3}$

Ovarian dysgenesis is related to the abnormal growth of ovary and occurs in $95 \%$ cases. This may lead to a delayed or lost of sexual development when the patient enters puberty. Incidence of patients who have spontaneous puberty signs at adolescent is $12 \%$. Patients usually develop amenorrhea. Several teenagers will experience 


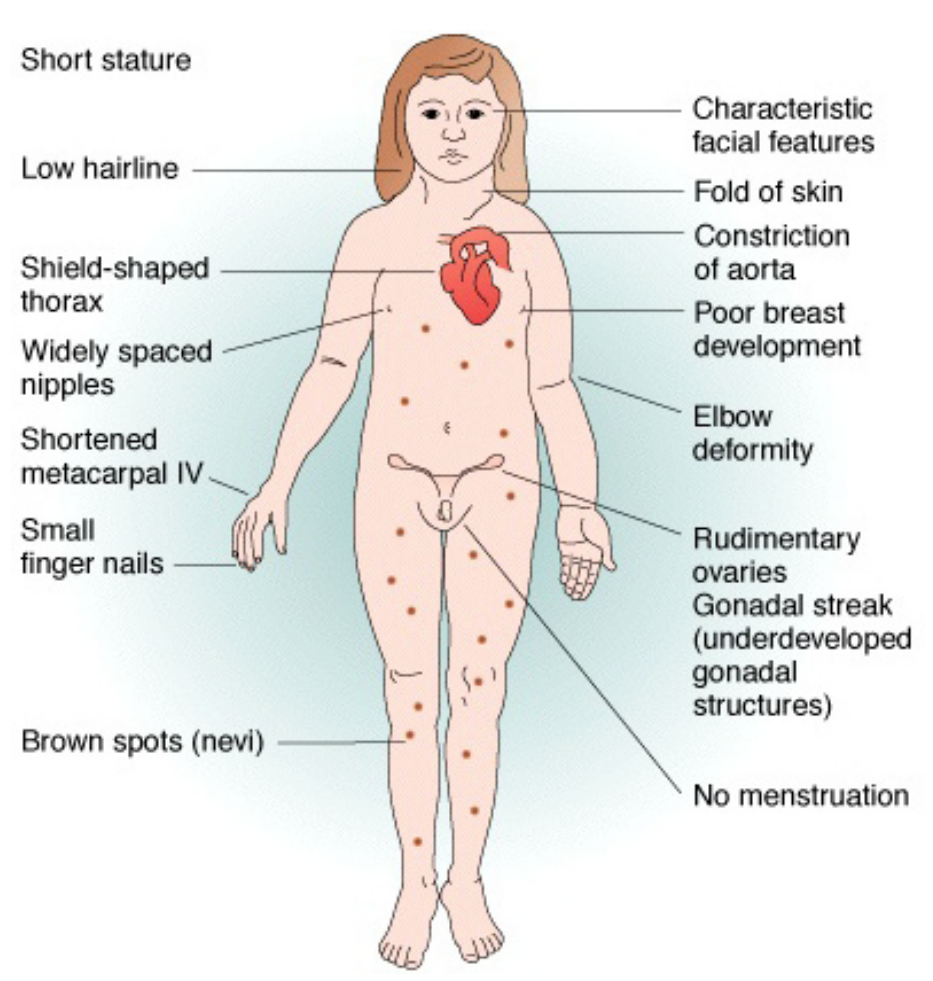

Figure 1. Characteristic of Turner syndrome. ${ }^{3}$

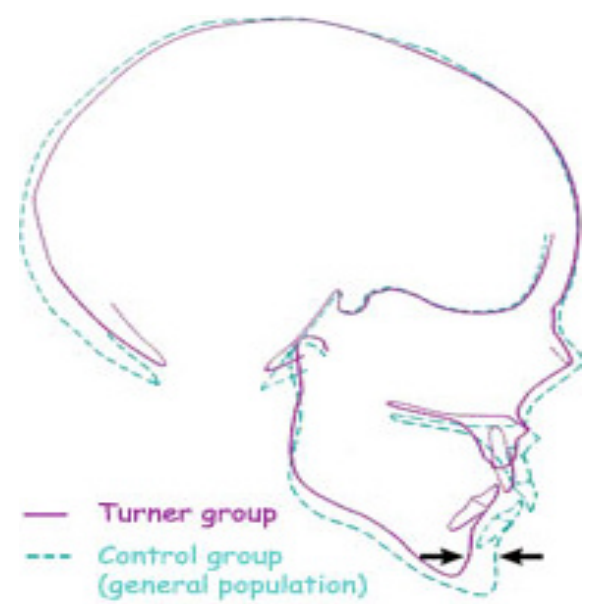

Figure 2. Comparative size of craniofacial in Turner syndrome and normal population. ${ }^{10}$

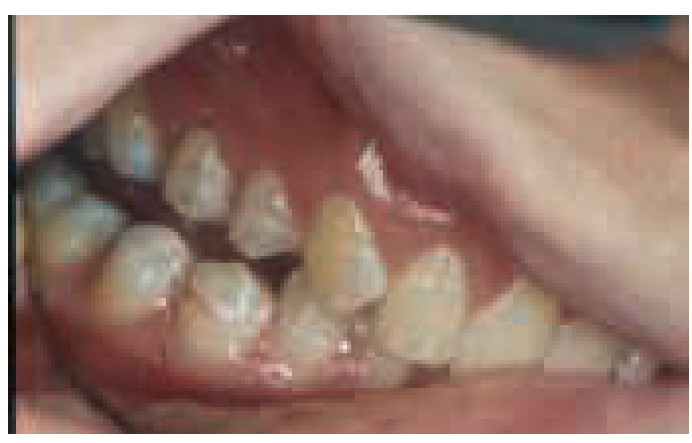

Figure 5. Enamel hypoplasia of Turner syndrome child. ${ }^{11}$

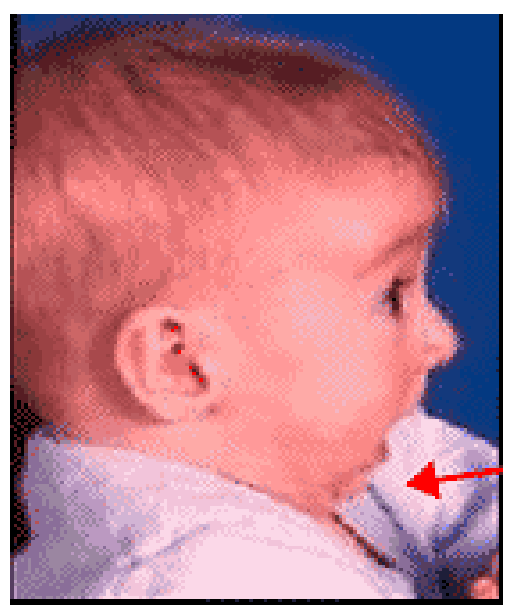

Figure 3. Patients' profile. ${ }^{3}$

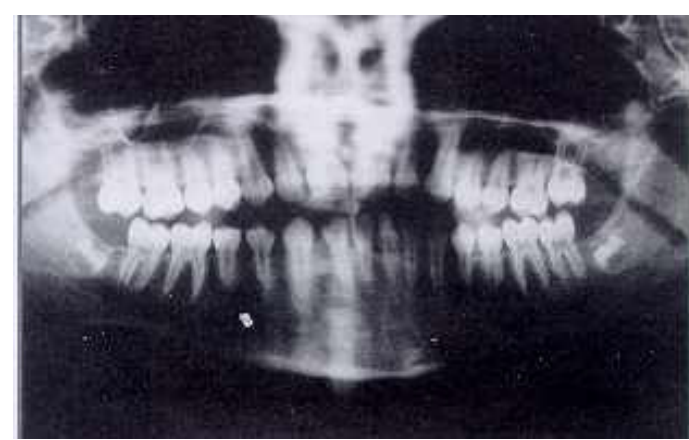

Figure 6. Panoramic radiograph of Turner syndrome at the age of 8 showed premature loss in primary dentition and premature eruption od permanent dentition. 


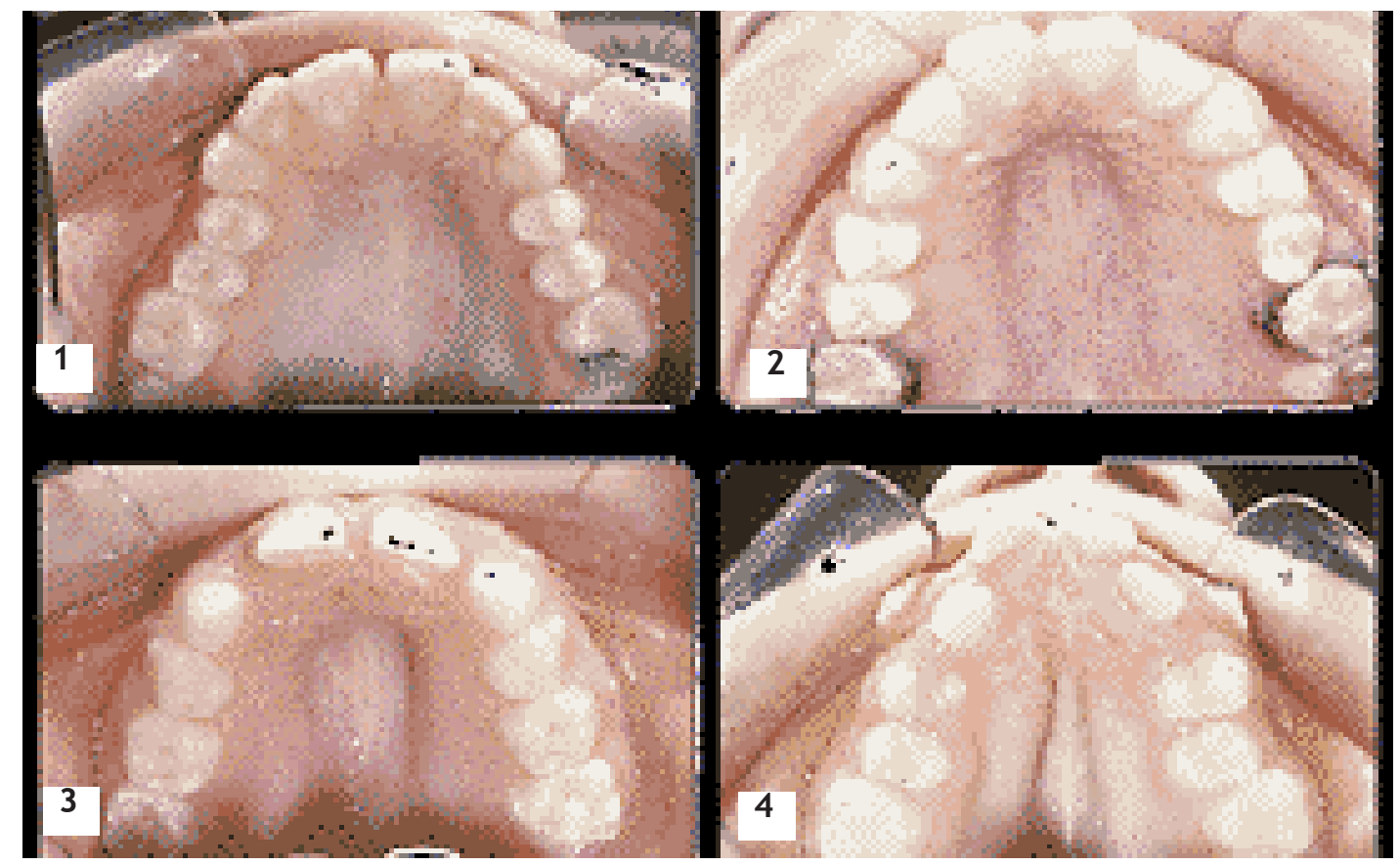

Figure 4. Palatal arch of Turner syndrome: (1) Normal; (2) Mild; (3) Moderate; (4) Severe. ${ }^{3}$

menstruation then there was a disturbance in the gonad which may lead to stop growth of ovary. Teenagers with spontaneous pubertal development have the risk of premature menopause. ${ }^{3}$

Growth and development of Turner syndrome is later than normal girl in almost all periods. Delayed in the increase of body height is the main reason for a parent to bring their undiagnosed child to seek for help. After birth, patients have a height of a few centimeters below normal compare to normal babies. The rate of increase body height is later after two years of age, leading to short stature. ${ }^{5}$

Turner syndrome is diagnosed by genetic examination, since it is a genetic disorder. Diagnostic test is done by examining chromosome pattern in the blood samples which called caryotiping. Prenatally, diagnosis can be done by amniocentesis. ${ }^{3}$

\section{Craniofacial growth and development of Turner syndrome}

Child with Turner syndrome has a different growth and development compare to normal child, generally. Bone formations undergo abnormal process. The abnormalities are delayed skeletal maturation, small bone, and reduced of Bone Mineral Density (BMD). Factors which affecting the abnormal growth and development are mainly cause by hormone and genetic. ${ }^{3}$
Craniofacial of Turner syndrome compare to normal child shows delayed development of cranium, reduced size of craniofacial complex, facial retrognathia, increased incisors overjet, reduced overbite, class II malocclusion, and abnormality of jaws relationships. ${ }^{6}$

Disturbance of bone growth in Turner syndrome is the result of skeletal dysplasia which may lead to short stature, slight ephyfiseal dysplasia, and abnormal bone formations. Skeletal dysplasia occurs because there is deficiency in the gene that brings SHOX. These also lead to delayed bone formation and short stature. ${ }^{1}$

SHOX protein is a group of cells which play an important role in chondrogenesis process. One of the reason why Turner syndrome child have short stature is because one or a part of $X$ chromosome is missing leading to the lost of active SHOX. ${ }^{3}$ Blocked chondrogenesis process may also block the growth of craniofacial, since the growth of skull and jaws insist of three main mechanism, which are the growth of cartilage, suture, and periosteal and endosteal. ${ }^{7}$

Cartilage growth in the skull is mainly occurs in basis cranii, nasal septal, and mandibular condyles. Cartilage growth at spheno-occipital synchondrosis may increase the antero-posterior dimension of basis cranii. Every area of cartilage growth plays an important role in the growth of 
head, especially in the first year of life. ${ }^{7}$

Gonadal dysplasia which occurs in Turner syndrome may lead to a deficiency in the secretion of estrogen hormone. The hormone is produce by ovary and stimulate the development of sex organs in females, breast, and variable secondary sex characteristics. It also affects to the growth and development of bone. Estrogen increased the osteoblastic activities and affects the growth of skeletal bone by causing the early union of ephysise with the body of long bones. ${ }^{8}$

In normal individuals, the secretion of estrogen hormone will reduce along with the increase of age and stops after menopause. Deficiency in estrogen level may result as reduced of osteoblastic activities in the bone, bone matrix, and the deposit of calcium and phosphate in the bone. ${ }^{8}$

Generally, Turner syndrome patients experience the deficiency of estrogen before, during, and after puberty. Estrogen in the prepuberty period function as in the increase of bone size which does not happened in Turner syndrome. Low secretion of estrogen may cause delayed bone growth. ${ }^{9}$

Study of 32 Turner syndrome children aged 7-16.7 compare to 33 normal girls aged 10.216.7 by Midtbo and Halse ${ }^{10}$ showed different size of calvaria and face. Calvaria and face in Turner syndrome girls showed a smaller size (Fig. 2). This morphology is characterized by flat cranial angle, shortened posterior cranial base, facial retrognathy, and rotation of jaws. These results showed a deviation in the pattern of the size and morphology of craniofacial in this syndrome which already exists since chilhood.

\section{Jaws growth and development of Turner syndrome}

Sixty percent of Turner syndrome patient have micrognathia. Generally, this occurs in mandible, thus showed a feature that lower jaw is retrognathia and upper jaw is normal or prognathia (Fig. 3). Another feature showed an abnormality in the growth of palate. This shows a high palatal arch in the form of $U$ or $V$ (Fig. 4). This case occurs in about $80 \%$ Turner syndrome and may lead to skeletal class II malocclusion. 3,11

\section{Dental implications of Turner syndrome}

Malocclusion is one of clinical sign of
Turner syndrome. Another dental implications are smaller size of tooth crown, abnormal quantity of roots, enamel hypoplasia (Fig. 5), thin dentine, posterior open bite, cross bite, premature eruption of permanent dentition, and delayed tooth maturation. ${ }^{11,12}$

Dental eruption in Turner syndrome girls usually 3.7 months faster than normal. Premature eruption in permanent dentition especially occurs in first molars at the age of 4 , normally at the age of 6 (Fig. 6). ${ }^{11,13}$

\section{Management of Turner syndrome}

Generally, Turner syndrome patients are under supervision by endocrinologist for routine check up and therapies. Therapy for Turner syndrome includes growth hormon therapy followed by estrogen injection. Growth hormone therapy is aimed to enhance linear growth. Estrogen therapy is necessary to make Turner syndrome girl more feminine, delay osteoporosis process, and stimulate the development of secondary sex characteristic. ${ }^{3}$

Other than main therapy, which are growth hormone and estrogen therapy, endocrinologist also give a supportive therapy. The later consist of anabolic steroid, antihypertension, vitamin and mineral, and thyroid therapy. ${ }^{14}$

\section{CONCLUSION}

Turner syndrome causes a disturbance in growth and development of the body which affects the growth and development of craniofacial complex. Growth hormone therapy gives a significant result in the growth and development of bone, but it does not correct the abnormalities in dental morphology and eruption. The management of craniofacial abnormalities in Turner syndrome can be managed by the cooperation of pedodontist, orthodontist, and endodontist.

\section{REFERENCES}

1. Sybert VP, McCauley E. Turner's syndrome. New England J Med 2004;351:1227-38.

2. Saenger P. Turner's syndrome. New England J Med 1996;355:1749-54.

3. Ross JL, Dennis-Feezle, Weber C. Turner syndrome: toward early recognition and 
improved outcomes. [cited 2005 Nov 28] Available from: http//www.medscape.com.

4. Ploof S. Turner's syndrome definition. [cited 2005 Nov 28]. Available from: http//www. turner's syndrome.htm.

5. Nielsen J, Naeraa RW. Turner's syndrome and Turner contact group. [cited 2005 Nov 28] Available from: http//www.aaa.dk.

6. Davenport ML. The effect of growth hormone on craniofacial growth and dental maturation in turner syndrome. The Angle orthodontist 2001;71(1):50-9.

7. Foster TD. A textbook of orthodontics. $3^{\text {rd }}$ ed. Jakarta: EGC; 1999. p. 1-20,68.

8. Guyton, Hall. Textbook of medical physiology. Jakarta: EGC; 1996.

9. Rubin K. Turner syndrome and osteoporosis: mechanisms and prognosis. Pediatrics
1998;102(2):481-5.

10. Midtbo M, Wisth PJ, Halse A. Craniofacial morphology in young patients with Turner syndrome. Eur J Orthod 1996;18(3):215-25.

11. Carter VL. The treatment complexities of Turner's syndrome patients (case studies) Dent Hygiene 2003;1(1).

12. Simmons KE. Growth hormone and craniofacial changes: preliminary data from studies in Turner's syndrome. Pediatrics 1999;104(4):1021-4.

13. Szilagyi A, Keszthelyi G, Nagy G, Madlena. Oral manifestations of patients with Turner syndrome. Oral Surg Oral Med Oral Pathol Oral Radiol Endod 2000;89(5):577-84.

14. Postellon D. Turner syndrome. [cited 2005 Nov 28]. Available from: http//www.emedicine. com. 